\title{
Correspondence: "Determinants of resilience among people who sustained spinal cord injury from the 2015 earthquake in Nepal"
}

\author{
Tayyebeh Ali-Abadi $^{1} \cdot$ Mohsen Soleimani $^{2}$ - Ali Fakhr-Movahedi ${ }^{3}$
}

Received: 15 February 2018 / Accepted: 5 March 2018 / Published online: 5 April 2018

(c) International Spinal Cord Society 2018

Initially we would like to thank Bhattarai et al. [1] for sharing their interesting findings about the determinants of resilience among people with spinal cord injury. Although their study provided valuable information about purposeful care delivery to people with spinal cord injury who are at risk for mental health problems, there are some concerns over outcome measurement and findings in the study. The concerns are explained as follows.

Pain intensity had been measured in the study using a numeric rating scale. However, instead of providing detailed information about the assessment of its reliability, the authors just noted that the reliability of the scale had been confirmed in an earlier study [2]. As random error may occur due to conditions that surrounded measurement and or individual differences of research sample, the estimation of reliability in a previous study is insufficient and reliability should be determined again in each new measurement [3].

Regression analysis is used to model the relationship between dependent variable $(\mathrm{Y})$ and explanatory variable (Xi), and the type of regression model depends on the type of distribution of Y. Usually, modeling can estimate the dependent based on explanatory variable predictors [4]. In fact, general linear model (GLM) univariate procedure provides an analysis for one dependent variable by one or more independent variables; and the GLM multivariate procedure are used for multiple dependent variable by one or more factors [5]. Now we do not know why the authors,

Ali Fakhr-Movahedi

alimovi49@semums.ac.ir

1 Nursing and Midwifery School, Semnan University of Medical Sciences, Semnan, Iran

2 Nursing Care Research Center, Nursing and Midwifery School, Semnan University of Medical Sciences, Semnan, Iran

3 Nursing Care Research Center, Nursing and Midwifery School, Semnan University of Medical Sciences, Semnan, Iran despite having a dependent variable, were used the multivariate analysis.

For each test, the legitimacy of the methods used to identify the cut-off scores is the main source of evidence for the validity of the instrument [6]. Cutting scores are usually used as the basis for deciding on needed treatments or further evaluations. It is necessary that the cut-off point reflects the similarity of scores in different cultural groups. Therefore, for translated instruments, a criterion validity estimation is necessary to determine the appropriate cutting point or to achieve higher consistency original instrument [7]. However, the cut-off point in this study was based on Rajan et al. [8] study in population of Indian children with intellectual disabilities. But, the concept of resilience has a multi-dimensional structure that varies with contextual conditions, time, age, and living circumstances [9]. Also, the experience of resilience varies according to the stages of life $[10,11]$ and changes over time as a person develops and interacts with environment [12]. Accordingly, despite the diversity in the cultural context of the Nepal's people, it would be better to determine cut-off point by receiver operating characteristic (ROC) curve analysis to show maximum specific and sensitive scores to provide costeffective services to people with true low resiliency.

Finally, as the study of Bhattarai et al. [1] provides valuable information about resiliency determinants among clients with spinal cord injuries; it is suggested that the above-mentioned points be considered in future studies in order to enhance more evidence-based results.

\section{Compliance with ethical standards}

Conflict of interest The authors declare that they have no conflict of interest. 


\section{References}

1. Bhattarai M, Maneewat K, Sae-Sia W. Determinants of resilience among people who sustained spinal cord injury from the 2015 earthquake in Nepal. Spinal Cord. 2018;56:78-83.

2. Krebs EE, Carey TS, Weinberger M. Accuracy of the pain numeric rating scale as a screening test in primary care. J General Intern Med. 2007;22:1453-8.

3. Waltz C, Strickland O, Lenz E. Measurement in nursing and health research. 4th ed. New York: Springer Publishing Company; 2010.

4. Alexopoulos EC. Introduction to multivariate regression analysis. Hippokratia. 2010;14(Suppl 1):23-8.

5. Hidalgo B, Goodman M. Multivariate or multivariable regression? Am J Public Health. 2013;103:39-40.

6. Holmes Finch W, Immekus JC, French BF. Applied psychometrics using SPSS and AMOS. Charlotte, NC: IAP; 2016.
7. Polit DF, Yang FM. Measurement and the measurement of change: a primer for the health professions. Philadelphia: Wolters Kluwer Health; 2015.

8. Rajan AM, John R. Resilience and impact of children's intellectual disability on Indian parents. J Intellect Disabil. 2017;21:315-24.

9. Singh K, Yu X-N. Psychometric evaluation of the ConnorDavidson Resilience Scale (CD-RISC) in a sample of Indian students. J Psychol. 2010;1:23-30.

10. Windle G, Bennett KM, Noyes J. A methodological review of resilience measurement scales. Health Qual life Outcomes. 2011;9:8.

11. Xie Y, Peng L, Zuo X, Li M. The psychometric evaluation of the Connor-Davidson resilience scale using a Chinese military sample. PLoS ONE. 2016;11:e0148843.

12. Southwick SM, Bonanno GA, Masten AS, Panter-Brick C, Yehuda R. Resilience definitions, theory, and challenges: interdisciplinary perspectives. Eur J Psychotraumatology. 2014;5:25338. 